\title{
14-3-3 gene characterization and description of a second 14-3-3 isoform in both Echinococcus granulosus and E. multilocularis
}

Received: 5 May 2003 / Accepted: 17 May 2004/Published online: 25 June 2004

(C) Springer-Verlag 2004

\begin{abstract}
Members of the 14-3-3 protein family have been identified as regulatory molecules in intracellular signaling pathways and cell cycle control. Previously, the first Echinococcus 14-3-3 isoform (E14-3-3.1) was isolated from E. granulosus and E. multilocularis metacestode stages. Hyperexpression of this isoform was claimed to be associated with non-restricted tumor-like growth of the E. multilocularis metacestode. In this report, we describe the characterization of a 14-3-3 cDNA from E. granulosus and E. multilocularis corresponding to a second isoform of this family, E14-3-3.2. The characterized 14-3-3 gene was interrupted by two introns whose sequence and positions were conserved in both Echinococcus species. The deduced amino acid sequence of E14-3-3.2 showed 88\% identity to the E14-3-3.1 isoform and $52 \%$ identity to a third Echinococcus isoform (E14-3-3.3) described by other authors. These findings, coupled to Southern blot analysis, suggest the presence of more than one 14-3-3 gene in Echinococcus. Phylogenenetically, the Echinococcus 14-3-3.1 and 14-3-3.2 isoforms appeared to cluster with zeta-type ("protumorigenic") 14-3-3 isoforms from closely related organisms, whereas the E14-3-3.3 isoform grouped with 14-3-3 epsilon isoforms. The presence of more than one 14-3-3 isoform might indicate isoform-specific roles in the different parasite stages of Echinococcus.
\end{abstract}

C. P. Nunes $(\bowtie) \cdot$ A. Zaha

Centro de Biotecnologia, Universidade Federal do Rio Grande do Sul, Caixa Postal 15005, RS 91501-970 Porto Alegre, Brazil

E-mail: paiva@adufrgs.ufrgs.br

Tel.: + 55-51-33166070

Fax: + 55-51-33167309

B. Gottstein · N. Müller

Institute of Parasitology, University of Berne, Switzerland

M. M. Siles-Lucas

Unidad de Parasitología, Facultad de Farmacia,

Universidad de Salamanca, Spain

\section{Introduction}

Echinococcosis is a worldwide zoonotic disease, with Echinococcus granulosus and E. multilocularis being the most studied species. The larval stage, the metacestode, develops in several mammalian intermediate host species, including humans, while the adult tapeworm generally develops mainly in dogs and foxes. The adult worm reproduces sexually, while asexual proliferation takes part inside the metacestode (Thompson 1995). One of the major physio-morphological differences between E. granulosus and E. multilocularis lies in the metacestode structure and growth behavior. More specifically, the E. granulosus metacestode is represented by an unilocular, fluid-filled, slowly-growing cyst, consisting of an inner germinal layer, a subsequent laminated layer and an external adventitial tissue, the latter produced by the host. The E. multilocularis metacestode includes basically the same tissue structures. It is, however, multivesiculated and exhibits infiltrative proliferation, tumor-like behavior and no restrictive host-tissue barrier (Siles-Lucas et al. 2001). For E. multilocularis, a putative relationship between the stage-specific overexpression of the parasite 14-3-3 protein and the nonrestricted growth of the E. multilocularis metacestode has been proposed (Siles-Lucas et al. 1998, 2001).

Initially discovered at high expression levels in the mammalian brain, the 14-3-3 proteins were later isolated from a wide range of eukaryotic organisms and cell types. In mammals, seven 14-3-3 isoforms have already been described. These are found differentially expressed in a variety of tissues and are associated with several key cell-cycle molecules, suggesting multifunctional roles for the 14-3-3 proteins (Aitken et al. 1992; Jones et al. 1995). Similar to what is described for the actin protein family, 14-3-3 isoform-specific sequences are conserved among eukaryotes (Wang and Shakes 1996; Rosenquist et al. 2000; Ferl et al. 2002).

The 14-3-3 proteins are also found in parasites, e.g. Schistosoma mansoni, in which three isoforms have 
already been described (Schechtman et al. 1995; McGonigle et al. 2001, 2002).

In Echinococcus, the presence of a second 14-3-3 isoform was suggested, as detected by immunoblot and immunolocalization in E. granulosus adult worms (SilesLucas et al. 2000). A putative third isoform from this cestode was later identified by Fernández et al. (2002). The presence of additional 14-3-3 isoforms in Echinococcus cannot be ruled out. Furthermore, 14-3-3 gene organization, intron-exon structure and genetic processing for each isoform are still unknown in this parasite genus.

In the present work, a polymerase chain reaction (PCR)-based approach was used to investigate both the genetic structure of the 14-3-3 gene(s) in E. granulosus and E. multilocularis and also to isolate and characterize other possible 14-3-3 isoforms from different parasite stages (adult worms, protoscoleces, metacestode tissue) of Echinococcus species.

\section{Materials and methods}

Parasite material and nucleic acid (DNA, RNA) extraction

Echinococcus granulosus protoscoleces and metacestode tissue (germinal layer) were obtained from naturally infected cows at local abattoirs in Rio Grande do Sul (Brazil). E. granulosus adult worms were kindly provided by Dr. Malgor (Unidad de Biología Parasitaria, Faculdad de Ciencias, Instituto de Higiene, Universidad de la Republica, Montevideo, Uruguay). E. multilocularis metacestode tissue samples (isolate KF5) were obtained either from experimentally infected mice or from in vitro culture-derived material, as described by Siles-Lucas et al. (1998).

E. multilocularis adult worms were kindly provided by Dr. P. Deplazes (Institute of Parasitology, University of Zurich, Switzerland). Total RNA from all the abovementioned samples was extracted using the Trizol reagent (Life Technologies, Basel, Switzerland) according to the manufacturer's instructions. Total genomic DNA from both parasite species was extracted by standard procedures, as described by McManus et al. (1985).

\section{Southern blot analysis}

For Southern analysis, $10 \mu \mathrm{g}$ of E. granulosus total genomic DNA was digested with EcoRI, HincII, HindIII, SalI or XhoI (Life Technologies, Rockville, Md.). After digestion, the respective products were separated by agarose electrophoresis in Tris-borate-EDTA (TBE) buffer, transferred onto positively charged Nylon membranes (Hybond- $\mathrm{N}+$; Amersham Biosciences, Little Chalfont, UK) by capillary action, dried and cross-linked at $80{ }^{\circ} \mathrm{C}$ for $2 \mathrm{~h}$. A specific DNA probe, consisting of the cDNA fragment corresponding to nucleotides 57-365 of the E. granulosus 14-3-3 isoform 2 isolated in this work, containing an internal HincII site, was obtained through PCR using the primer pair 12AA (degenerated; 5'-GCGAARCTTGCBGARCARGC-3') and $65 \mathrm{U}\left(5^{\prime}\right.$-GCTTCAGCACCAATTTTCTC- $\left.3^{\prime}\right)$. This 308 -bp fragment was radioactively labeled with $\alpha 32-$ dCTP, using the Random primer DNA labeling system (Life Technologies, Rockville, Md.) according to the manufacturer's instructions. Probe hybridization on the membrane containing the digested E. granulosus genomic DNA was carried out at $60{ }^{\circ} \mathrm{C}$ overnight, followed by four consecutive 15 -min washes in $5 \times, 2 \times, 1 \times$ and $0.5 \times$ $0.3 \mathrm{M}$ standard sodium citrate buffer plus $0.1 \%$ SDS at $40{ }^{\circ} \mathrm{C}$.

\section{RT-PCR and PCR amplification}

First-strand cDNA was synthesized from E. granulosus and E. multilocularis adult worms, protoscoleces and metacestode tissue using Superscript reverse transcriptase (Life Technologies, Basel, Switzerland) or the 5'RACE/3'RACE kit (Boehringer, Mannheim, Germany) as recommended by the manufacturer. The cDNAs were subsequently subjected to PCR reactions, using the 5'RACE anchor primer (Boehringer, Mannheim, Germany) and the Echinococcus 14-3-3 E2U specific primer (5'-CTCAATCAGAACCACGACAG-3'). For Echinococcus genomic DNA amplification, PCR reactions were done with specific iso2 (5'-ATGGCAGCTATCACCTCTTGG-3') and E2U primers. All PCR reactions were performed with $100 \mathrm{mmol}$ of each dNTP, $100 \mathrm{ng}$ of each primer and 1 unit of Taq DNA polymerase, plus respective buffer (Cenbiot, UFRGS. Porto Alegre, Brazil), in a final volume of $50 \mu$ l. Reactions were run for 35 cycles of $94{ }^{\circ} \mathrm{C}$ for $40 \mathrm{~s}$, $50{ }^{\circ} \mathrm{C}$ for $1 \mathrm{~min}$ and $72{ }^{\circ} \mathrm{C}$ for $1 \mathrm{~min}$, with a final extension step of $72{ }^{\circ} \mathrm{C}$ for 5 min.

\section{Cloning and sequencing of specific DNA fragments}

PCR-amplified products were separated by electrophoresis through TBE-agarose gels. Corresponding bands were excised from the gel and purified using the GFXTM-PCR-DNA and Gel band purification kit (Amersham Biosciences), following the manufacturer's instructions. Purified PCR amplicons were directly sequenced (see next section) or cloned into the pGEM-T vector (Promega, Madison, USA), again following the manufacturer's instructions. Corresponding ligation reactions were used for the transformation of Escherichia coli XL1-Blue competent cells, according to a protocol described by Sambrook et al. (1989). Selected recombinant colonies were grown and corresponding recombinant plasmids were extracted using the PeqLab kit (Stratagene, La Jolla, Calif.). PCR products and recombinant pGEM-T vectors were either automatically sequenced (Microsynth, Basel, Switzerland), or 
manually sequenced by the dideoxy chain-termination method (Sanger et al. 1977) using the Thermo Sequenase radiolabeled terminator cycle sequencing kit (Amersham Biosciences) and the Model SA adjustable sequencing gel electrophoresis system (Life Technologies, Rockville, Md.) according to the manufacturers' instructions. Primers T7 and M13 were used for pGEM-inserted sequences and primers iso2 and E2U for purified PCR products.

Alignments, phylogenetic analysis and gene structure comparison

The specific 14-3-3 sequences from both E. granulosus and E. multilocularis genomic DNA and cDNA fragments obtained during our experiments, together with those from the same species available in GenBank (accessions AF529418, AF529419, BF643012), were aligned using the CLUSTAL X (Thompson et al. 1997) and TEXshade (Beitz 2000) computer programs. The putative translation of the above-mentioned sequences was used to construct a second alignment (see Fig. 1). In order to perform a phylogenetic analysis, a third alignment was done with the three Echinococcus putative protein sequences corresponding to isoforms 1,2 and 3,

Fig. 1 Alignment of Echinococcus granulosus and E. multilocularis 14-3-3 predicted amino acid sequences. The alignment was generated using the ClustalX and TEXshade computer programs. Isoform 1.1 E. granulosus metacestode tissue, Isoform 1.2 E. multilocularis metacestode tissue, Isoform 2.2 E. granulosus adult worms, Isoform $2.3 \mathrm{E}$. multilocularis adult worms, Isoform 2.1 E. granulosus and E. multilocularis adult worms, Isoform 3 E. granulosus protoscoleces, Consensus consensus sequence. Identical amino acids are shaded in black. Boxes correspond to similar regions. Dots indicate gaps in the generated alignment. Upper case indicates conserved residues in all aligned sequences, lower case indicates conserved residues in $60-80 \%$ of the aligned sequences including also those 14-3-3 protein sequences from other organisms used in the phylogenetic analysis performed by Wang and Shakes (1996), plus the GenBank-available sequences of the 14-3-3 protein from Schistosoma (see Fig. 4). A neighbor-joining distance tree was obtained from the full alignment of the core region of different 14-3-3 isoforms from several organisms, using the MEGA ver. 2.1 program (Kumar et al. 2001).

Genomic DNA sequences from both E. granulosus and E. multilocularis, compared with the corresponding gene sequences in Caenorhabditis elegans (GenBank Z73910 and Z66564), Plasmodium knowlesi (GenBank AF065986) and P. falciparum (GenBank AF065987), were used for the construction of a gene physical structure map (see Fig. 2), as described by Voigt et al. (2000) and DeLille et al. (2001).

\section{Results}

Cloning, sequencing and sequence analysis

Sequencing of 14-3-3 cDNAs from Echinococcus granulosus and E. multilocularis obtained by PCR showed a 774-bp open reading frame for adult worms, protoscoleces and metacestode tissue, subsequently called isoform 2 (E14-3-3.2). Putative translation of sequences provided three proteins of 248 amino acids, which were identical, except for single substitutions (at position 187 in isoform 2.3 and position 241 in isoform 2.2; see Fig. 1). The three proteins showed the characteristic motifs of the 14-3-3 family (Wang and Shakes 1996). The E14-3-3.2 sequence presented minor differences from those previously published for the metacestode stage of both species (Siles-Lucas et al. 2001; GenBank AF207904, U63643), subsequently called Echinococcus 14-3-3 isoform 1 (E14-3-3.1). It also presented major

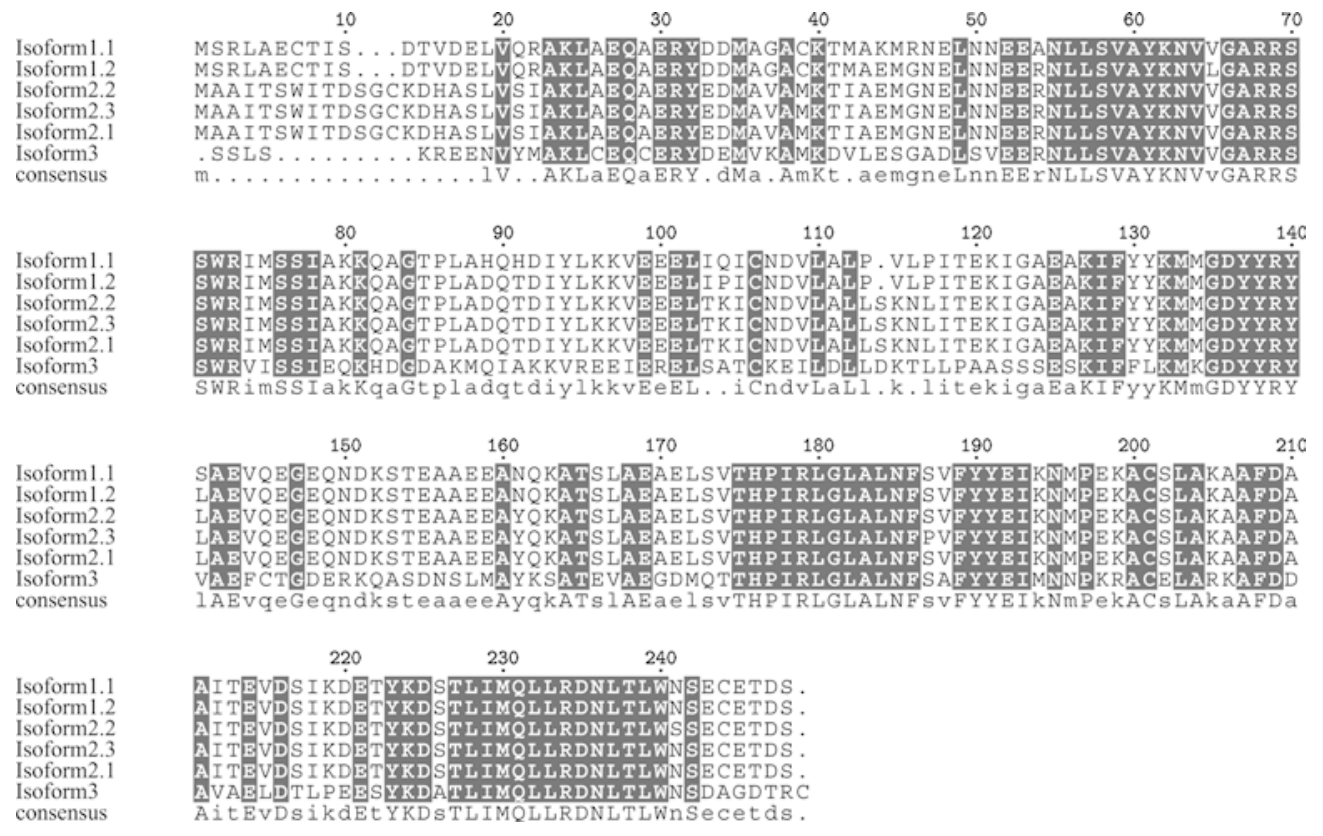


Fig. 2 Physical structure of 143-3 genes. C.e. ftt-1 and C.e. ftt2 Caenorhabditis elegans (GenBank Z73910, Z66564), E.g. 14-3-3.2 E. granulosus, E.m. 14-3-3.2 E. multilocularis, P.k. 14-3-3Plasmodium knowlesi (GenBank AF065986), P.f. 143-3 P. falciparum (GenBank AF065987). Boxes represent exons while lines represent introns. Numbers indicate amino acid positions at the beginning and the end of each exon
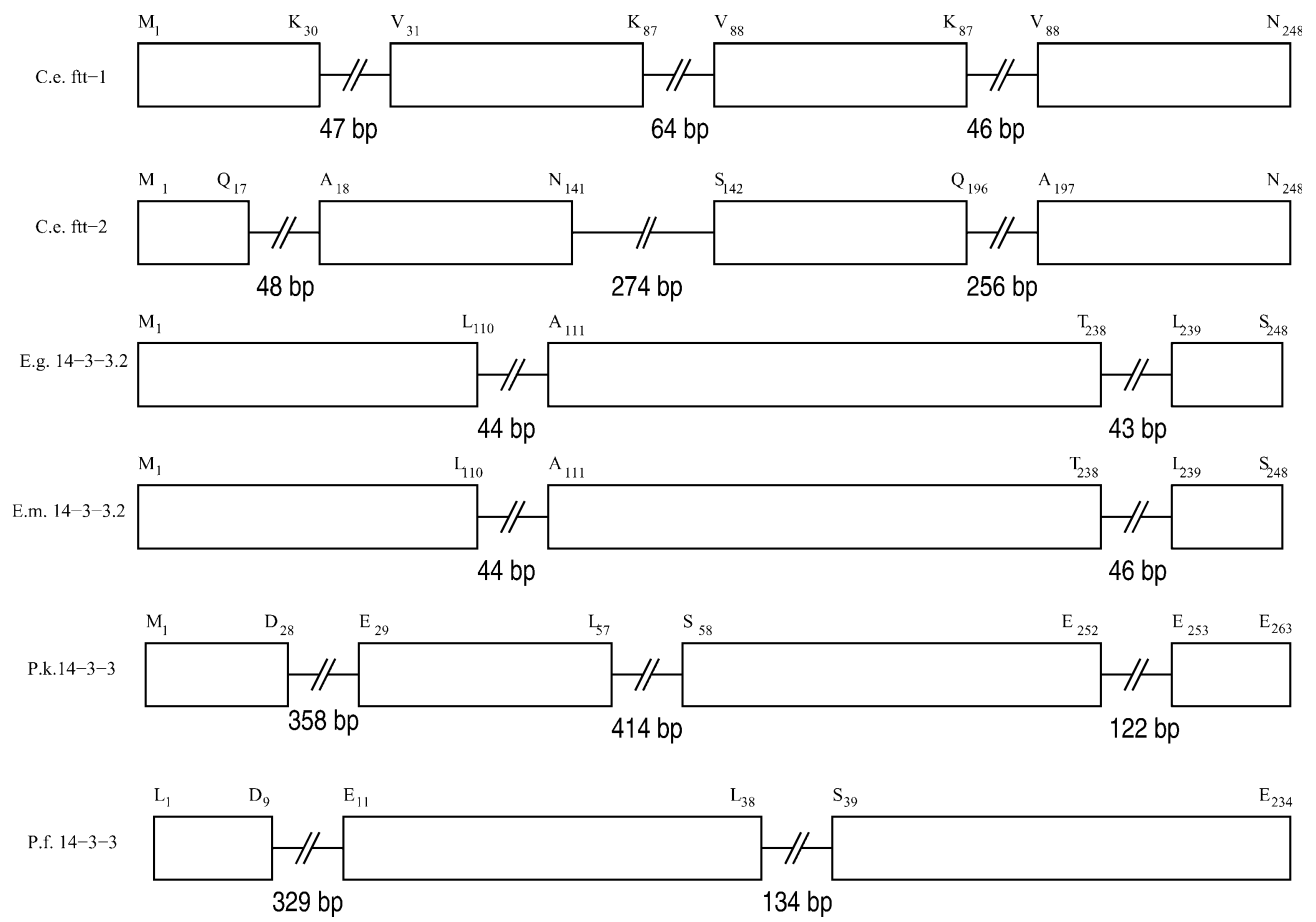

differences with a third, $5^{\prime}$-end truncated sequence, isolated from E. granulosus protoscoleces (Fernández et al. 2002) and subsequently called Echinococcus 14-3-3 isoform 3 (E14-3-3.3, GenBank BF643012; see Fig. 1).

The following differences were observed regarding both composition and length of the sequences:

1. E14-3-3.2 presented four additional amino acids when compared with E14-3-3.1.

2. The N-terminal region was the most divergent between both above-mentioned sequences.

3 . The overall identity between the isoforms, compared with isoform 1.1 (from E. multilocularis metacestode tissue) were $95 \%$ (isoform 1.2), $88 \%$ (isoforms 2.1, 2.2, 2.3) and 52\% (isoform 3; see Fig. 1).

To analyze the Echinococcus 14-3-3 genomic DNA sequences, PCR was carried out using the specific primers iso2 and E2U on total DNA from E. granulosus and E. multilocularis and the 14-3-3 genomic sequences from both species obtained here proved to be very similar ( $97 \%$ identity). The genetic structure of both sequences was compared with homologous genes in other organisms. As shown in Fig. 2, the genomic sequences from both Echinococcus species contained three exons, interrupted by two introns which were highly conserved in their positions and sequences. Exon-intron boundaries, which lay outside functional domains, were found to be conventional in both species (data not shown).

\section{Southern analysis}

To verify 14-3-3 gene copy numbers in Echinococcus, total DNA from E. granulosus was digested with several restriction enzymes, transferred to a Nylon membrane and hybridized with an Echinococcus 14-3-3-specific probe (see Materials and methods). DNA digested with EcoRI, SalI and XhoI, which do not cut within the known Echinococcus 14-3-3 cDNA and genomic DNA, resulted in seven, two and two hybridization bands with different intensities, respectively (see Fig. 3). Corresponding patterns with HincII and HindIII, which putatively cut once each inside the E. granulosus genomic DNA sequence (although at sites that lay either before or after the sequence of the probe used for hybridization; data not shown), exhibited six and four hybridization bands with different intensities, respectively.

Phylogenetic analysis of the Echinococcus 14-3-3 protein

Putative sequences from the E14-3-3.1, E14-3-3.2 and E14-3-3.3 protein isoforms were aligned with selected homologous sequences from other organisms (see Materials and methods). This alignment was further used for the construction of a phylogenetic tree, showed in Fig. 4. The Echinococcus 14-3-3.1 and 14-3-3.2 sequences clustered in a separate group from the Echinococcus 14-3-3.3 isoform. Isoforms 1 and 2 clustered with 14-3-3 sequences from eukaryotes (mostly mammals), including two Schistosoma 14-3-3 sequences. The third Echinococcus isoform integrated within the group corresponding to 14-3-3 sequences from Schistosoma, described as the epsilon isoform.

The sequence data reported herein have been deposited in GenBank under accessions AF529418, AF529419, AF529420. 


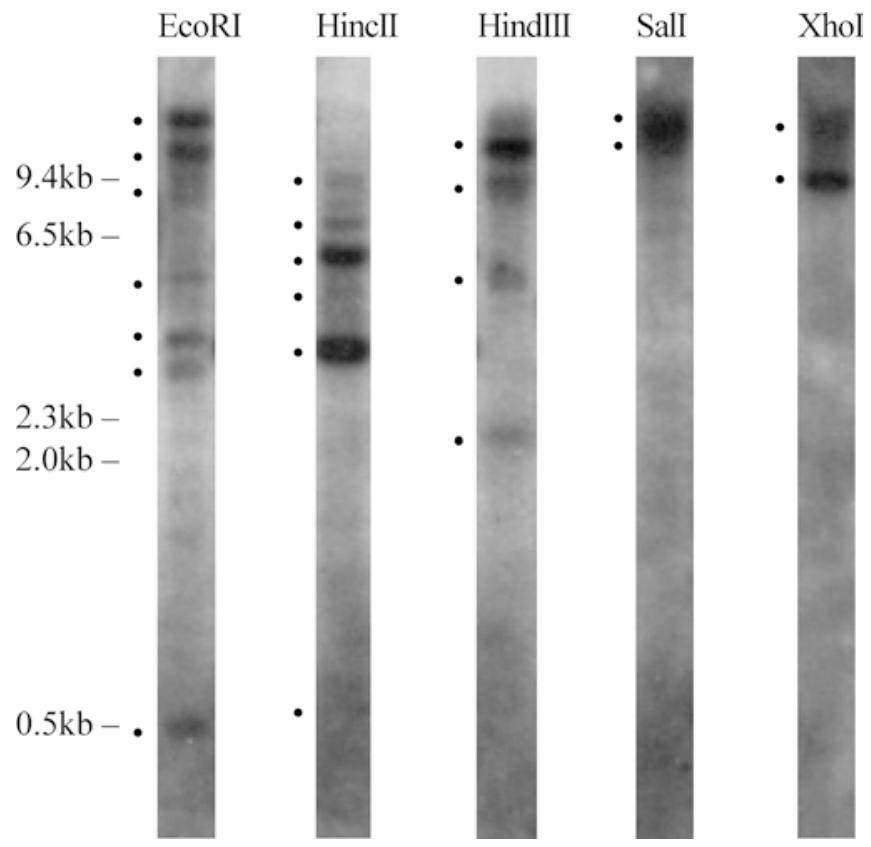

Fig. 3 Southern analysis of E. granulosus genomic DNA. Total DNA $(10 \mu \mathrm{g} /$ well $)$ was digested with EcoRI, HincII, HindIII, SalI and XhoI. A cDNA fragment corresponding to nucleotides 57-365 of the coding sequence from the E. granulosus adult worm 14-3-3 isoform 2 (see Fig. 1) was used as probe. Positions of DNA size markers (Amersham Biosciences phage $\lambda$ digested with HindIII) are indicated at the left of the figure. Corresponding hybridization signals in each well are marked with dots

\section{Discussion}

The 14-3-3 proteins have been described as key molecules in eukaryotic cell biology (Aitken et al. 1992). These proteins were present in several isoforms in the same organism, resulting in the presence of isoforms or counterpart (orthologous) sequences (Wang and Shakes 1996). Thus, one specific isoform of a defined organism shared higher identities with the corresponding isoform in other organisms than with other isoforms from the same organism.

The presence of several 14-3-3 isoforms within the same organism seems to be a general rule (Rosenquist et al. 2000; McGonigle et al. 2002). Recent findings suggest that differences between isoforms can reflect a cell-specific localization and/or a distinct functionality (Rosenquist et al. 2000). In Echinococcus, a first 14-3-3 isoform expressed in metacestode tissues was described, and its over-expression was related with the non-restricted growth potential of the E. multilocularis metacestode (Siles-Lucas et al. 1998; E14-3-3.1). Later, the presence of a second isoform was suggested, functionally related with metabolic (excretory/secretory) processes in Echinococcus adult worms (Siles-Lucas et al. 2001). Finally, a third isoform was isolated and sequenced from E. granulosus (Fernández et al. 2002; E14-3-3.3), but no putative functionality has so far been assigned to this last isoform.
We now report the identification and isolation of the second Echinococcus 14-3-3 isoform (called here E14-3-3.2), common to adult worms, protoscoleces and metacestode tissue from both E. granulosus and E. multilocularis. E14-3-3.2 sequences showed differences when compared with both E14-3-3.1 (88-86\% identity) and E14-3-3.3 (52\% identity). The percentage of identity between these latter two Echinococcus isoforms is smaller than that usually observed between different species in a given 14-3-3 isoform family, although higher than between different isoforms from the same species (Rosenquist et al. 2000). Similarly, isoforms ftt- 1 and ftt2 from Caenorhabditis elegans showed $90 \%$ similarity (Wang and Shakes 1997). Despite this high similarity, both sequences represent separate isoforms, which differ, among other details, in their expression patterns.

Both E. granulosus E14-3-3.1 and E14-3-3.2 isoforms share higher similarities with the corresponding isoform in E. multilocularis than with each other. This could indicate that the duplication event occurred before the separation of the two species.

The nucleotide alignment of isoforms E14-3-3.1 and E14-3-3.2 with a third, 5'-end truncated 14-3-3 isoform isolated from E. granulosus (Fernández et al. 2002) showed much lower similarities (approximately 50\%; data not shown). Thus, E14-3-3.3 corresponds to a distinct 14-3-3 isoform in Echinococcus, as it fulfills the expected requirements (Rosenquist et al. 2000).

The coding region of the Echinococcus 14-3-3 gene characterized in the present work corresponds to the E14-3-3.2 isoform, interrupted by two introns. We could not find, using the same PCR-based strategy, a genomic counterpart for isoforms 1,3 or others. This could be attributed to a high E14-3-3.1 specificity of the primers used in our approach.

Introns 1 and 2 were found in conserved positions when E. granulosus and E. multilocularis 14-3-3 genes were compared, although corresponding sequences were slightly different $(95 \%$ and $89 \%$ identity, respectively). The 14-3-3 intron sequences of the 14-3-3 genes described from Plamodium falciparum, P. knowlesi (AlKhedery et al. 1999) and C. elegans (Wang and Shakes 1997) showed no significant identity or similarity regarding size, position or sequence with those in the Echinococcus 14-3-3 gene found during our experiments. Lack of 14-3-3 intron conservation between different species has been found by other authors when comparing different isoforms (e.g., De Vetten and Ferl 1994), although clear similarities were found when comparing non-coding sequences from the same 14-3-3 isoform obtained from different organisms (Muratake et al. 1996; Tokooya et al. 2002). Unfortunately, sequences which could represent orthologous genes when compared with the Echinococcus 14-3-3 gene were not available.

The number of E. granulosus 14-3-3 genes was assessed by Southern blot. Corresponding results show that the E. granulosus genome contains more than one 14-3-3 gene, as DNA restriction with enzymes that do 
Fig. 4 Phylogenetic tree of selected 14-3-3 proteins (see Materials and methods), showing the positions of the Echinococcus 14-3-3 proteins in bold. The aligned sequences were analyzed by the neighborjoining distance method, using the MEGA ver. 2.1 program. Bootstrap values are indicated at each branch

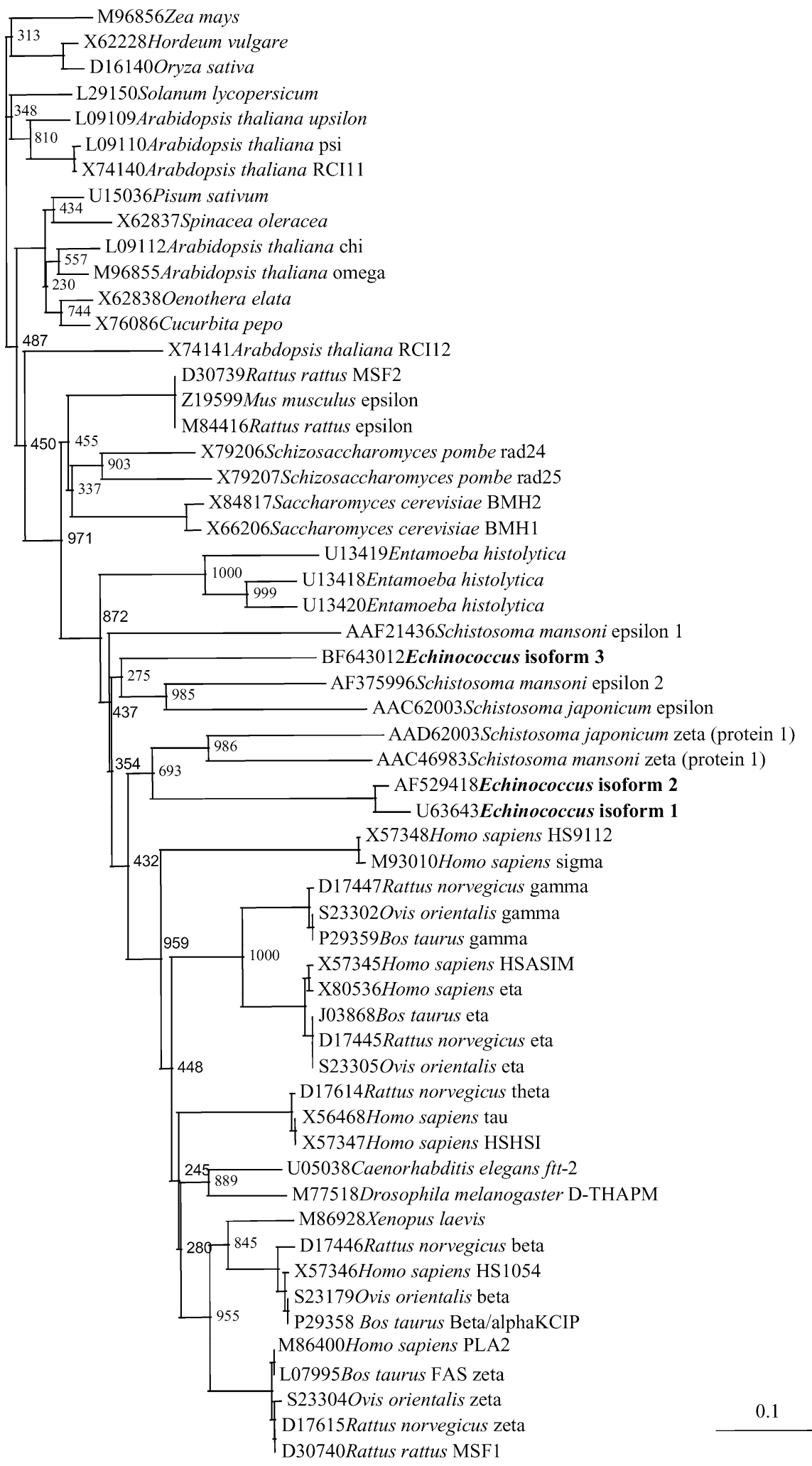

not cut in any of the Echinococcus 14-3-3 sequences known to date (Siles-Lucas et al. 1998; Fernández et al. 2002; present work) resulted in more than one hybridization signal, although the signals differed in their intensity. These results are in agreement with those found in the literature (Wang and Shakes 1996;
Rosenquist et al. 2000; Ferl et al. 2002), indicating the common occurrence of several 14-3-3 genes in other organisms. A discrepancy between the present results and those obtained before (showing the presence of a single 14-3-3 gene in E. multilocularis; Siles-Lucas et al. 1998) could be attributed to the use of different probes 
and respective specificity and/or differences in the stringency of the reaction.

The three E14-3-3 isoforms (protein sequences) from Echinococcus were used here in a phylogenetic approach to examine their evolution and to look for possible orthologous sequences in other organisms. The resulting phylogenetic tree shows that the Echinococcus 14-3-3.1 and 14-3-3.2 isoforms clustered in a distinct group, together with two Schistosoma sequences already described as zeta-like isoforms (McGonigle et al. 2002). Thus, our results confirm the close relationship of the E14-3-3.1 isoform with zeta-type ("pro-tumorigenic") 14-3-3 isoforms from other organisms already pointed out by Siles-Lucas et al. (2001). The position of the corresponding branch, in a separate cluster from the 143-3 isoforms other than epsilon, suggest that these zetalike parasite 14-3-3 proteins diverged earlier from the epsilon isoform than in other organisms. The third Echinococcus 14-3-3 isoform (E14-3-3.3) clearly grouped with the epsilon isoforms described for Schistosoma mansoni and S. japonicum (McGonigle et al. 2002). Thus, the E14-3-3.3 sequence represents the epsilon isoform in Echinococcus.

In summary, we found the presence of an additional 14-3-3 protein isoform and its corresponding gene (E143-3.2) in Echinococcus, which contained two introns. Comparison between the three Echinococcus isoforms and 14-3-3 proteins from other organisms showed that isoforms E14-3-3.1 and E14-3-3.2 lie in the zeta-like group, while E14-3-3.3 represents the epsilon isoform in this parasite. It will be of interest now to define possible differences in the functionality and localization of these three Echinococcus isoforms and their role in parasite survival and development within the host.

Acknowledgements This work was supported by PADCT (620081/ 95-3), CNPq, FAPERGS, the Swiss National Science Foundation (Project 31-63615.00) and the Ministry of Science and Technology in Spain (Project AGL2002-00644). M.S.L. is the holder of a "Ramón y Cajal" contract. We acknowledge the financial contributions made by the Interreg II project BWA 30.027 and the EU EchinoRisk project QLK2-CT-2001-01995 (BBW 00.0586-1) and also thank J.J. Arenzon for a critical reading of the manuscript and A.P. Nunes for help with the figures.

\section{References}

Aitken A, Collinge DB, Heusden BP van, Isobe T, Roseboom PH, Rosenfeld G, Soll J (1992) 14-3-3 proteins: a highly conserved, widespread family of eukaryotic proteins. Trends Biochem Sci 17:498-501

Al-Khedery B, Barnwell JW, Galinski MR (1999) Stage-specific expression of 14-3-3 in asexual blood-stage Plasmodium. Mol Biochem Parasitol 102:117-130

Beitz E (2000) TEXshade shading and labeling multiple sequence alignments using LaTeX2E. Bioinformatics 16:135-139

DeLille JM, Sehnke PC, Ferl RJ (2001) The Arabidopsis 14-3-3 family of signaling regulators. Plant Physiol 126:35-38

De Vetten NC, Ferl RJ (1994) Two genes encoding GF14 (14-3-3) proteins in Zea mays. Structure, expression, and potential regulation by the G-box binding complex. Plant Physiol 106:15931604
Ferl RJ, Manak MS, Reyes MF (2002) The 14-3-3s. Genome Biol 3:3010.1-3010.7

Fernández C, Gregory WF, Loke P, Maizels RM (2002) Fulllength-enriched cDNA libraries from Echinococcus granulosus contain separate populations of oligo-capped and trans-spliced transcripts and a high level of predicted signal peptide sequences. Mol Biochem Parasitol 122:171-180

Jones DH, Ley S, Aitken A (1995) Isoforms of 14-3-3 protein can form homo- and heterodimers in vivo and in vitro: implications for function as adapter proteins. FEBS Lett 368:55-58

Kumar S, Tamura K, Jakobsen IB, Nei M (2001) MEGA2: molecular evolutionary genetics analysis software. Bioinformatics 17:1244-1245

McGonigle S, Beall MJ, Feeney EL, Pearce EJ (2001) Conserved role for 14-3-3 epsilon downstream of type I TGF beta receptors. FEBS Lett 490:65-69

McGonigle S, Loschiavo M, Pearce EJ (2002) 14-3-3 proteins in Schistosoma mansoni: identification of a second epsilon isoform. Int J Parasitol 32:685-693

McManus DP, Knight M, Simpson AJ (1985) Isolation and characterisation of nucleic acids from the hydatid organisms, Echinococcus spp (Cestoda). Mol Biochem Parasitol 16:251-266

Muratake T, Hayashi S, Ichikawa T, Kumanishi T, Ichimura Y, Kuwano R, Isobe T, Wang Y, Minoshima S, Shimizu N, Takahashi Y (1996) Structural organization and chromosomal assignment of the human 14-3-3 eta chain gene (YWHAH). Genomics 36:63-69

Rosenquist M, Sehnke P, Ferl RJ, Sommarin M, Larsson C (2000) Evolution of the 14-3-3 protein family: does the large number of isoforms in multicellular organisms reflect functional specificity? J Mol Evol 51:446-458

Sambrook J, Fritsch EF, Maniatis T (1989) Molecular cloning: a laboratory manual, $2^{\text {nd }}$ edn. Cold Spring Harbor Laboratory Press, Cold Spring Harbor, N.Y.

Sanger R, Nicklen S, Coulson AX (1977) DNA sequencing with chain-terminating inhibitors. Proc Natl Acad Sci USA 74:54635467

Schechtman D, Ram D, Tarrab-Hazdai R, Arnon R, Schechter I (1995) Stage-specific expression of the mRNA encoding a 14-33 protein during the life cycle of Schistosoma mansoni. Mol Biochem Parasitol 73:275-278

Siles-Lucas M, Felleisen RS, Hemphill A, Wilson W, Gottstein B (1998) Stage-specific expression of the 14-3-3 gene in Echinococcus multilocularis. Mol Biochem Parasitol 91:281-293

Siles-Lucas M, Nunes CP, Zaha A, Breijo M (2000) The 14-3-3 protein is secreted by the adult worm of Echinococcus granulosus. Parasite Immunol 22:521-528

Siles-Lucas M, Nunes CP, Zaha A (2001) Comparative analysis of the 14-3-3 gene and its expression in Echinococcus granulosus and Echinococcus multilocularis metacestodes. Parasitology 122:281-287

Thompson JD, Gibson TJ, Plewniak F, Jeanmougin F, Higgins DG (1997) The ClustalX windows interface: flexible strategies for multiple sequence alignment aided by quality analysis tools. Nucleic Acid Res 24:4876-4882

Thompson RCA (1995) Biology and systematics of Echinococcus. In: Thompson RCA, Lymbery AJ (eds) Echinococcus and hydatid disease. CAB International, Wallingford, pp 1-50

Toyooka K, Muratake T, Watanabe H, Hayashi S, Ichikawa T, Usui H, Washiyama K, Kumanishi T, Takahashi Y (2002) Isolation and structure of the mouse 14-3-3 eta chain gene and the distribution of 14-3-3 eta mRNA in the mouse brain. Brain Res Mol Brain Res 100:13-20

Voigt J, Liebich I, Wostemeyer J, Adam KH, Marquardt O (2000) Nucleotide sequence, genomic organization and cell-cycledependent expression of a Chlamydomonas 14-3-3 gene. Biochim Biophys Acta 1492:395-405

Wang W, Shakes DC (1996) Molecular evolution of the 14-3-3 protein family. J Mol Evol 43:384-398

Wang W, Shakes DC (1997) Expression patterns and transcript processing of $f t t-1$ and $f t t-2$, two C. elegans 14-3-3 homologues. J Mol Biol 268:619-630 